\title{
Lattices of paths: Representation theory and valuations
}

\author{
Luca Ferrari and Emanuele Munarini
}

\begin{abstract}
We study some distributive lattices arising in the combinatorics of lattice paths. In particular, for the Dyck, Motzkin and Schröder lattices we describe the spectrum and we determine explicitly the Euler characteristic in terms of natural parameters of lattice paths.

AMS 2000 SUBJECT ClASSIFICATIONS: Primary 06D05; secondary 06A07. Keywords AND Phrases: Dyck paths, Dyck lattices, Motzkin paths, Motzkin lattices, Schröder paths, Schröder lattices, Dyck-like lattices, Young lattices, Euler characteristic, finite distributive lattices.
\end{abstract}

\section{Introduction}

The set of all lattice paths of equal length with steps of a prescribed kind (usually starting from the origin and ending on the $x$-axis with respect to a fixed Cartesian coordinate system) can always be ordered by containment. More precisely, we say that $\gamma_{1} \leq \gamma_{2}$ when $\gamma_{1}$ lies weakly below $\gamma_{2}$. In several cases, the resulting poset has the structure of a distributive lattice, as for Dyck, Motzkin and Schröder paths. Some conditions that guarantee to have a distributive lattice are given in [13], where there is also a first attempt to provide a systematic classification of these posets of paths. Some of these lattices turn out to be isomorphic with the lattices arising from other well known structures $[3,4,12]$. For instance, the lattice generated by all Dyck paths of length $n$ is isomorphic to the dual of the Young lattice associated with the staircase partition $(n, n-1, \ldots, 2,1)$, as proved in [24] or in [22] where a different combinatorial interpretation is provided. The language of lattice paths, however, gives a geometric flavor to the subject which allows to express several properties in a more fascinating way. Moreover, the study of lattices in terms of paths is a growing area of research, as witnessed by the investigation of a generalization of Dyck lattices appearing in [19] or by the deep study of the properties of Dyck lattices developed in [20].

In the present paper we consider essentially two kinds of questions concerning lattices of paths. The first topic concerns the representation theory of lattices of Dyck-like paths, i.e. paths defined as the ordinary Dyck paths except for the fact that they use up steps of the form $(1, a)$ and down steps 
of the form $(1,-b)$, where $a$ and $b$ are two assigned positive integers. Specifically, we obtain a representation theorem for these lattices describing explicitly their spectrum. We also prove that these lattices can be described as dual of Young lattices associated to a given partition. Then we review some results scattered in the literature and, in particular, for the Dyck lattices we observe some elementary properties which will be extensively used in the rest of the paper.

The second topic concerns the Euler characteristic of lattices of paths. The Euler characteristic is a classical invariant measure which plays an important role, for instance, in combinatorial geometry [16] and in geometric probability [15]. The combinatorial interest of the Euler characteristic lies in its deep relation with the Möbius function [18] (see also [17]). In the present paper, we give a general technique to determine the Euler characteristic of Dyck-like lattices, which generalize in a natural way the ordinary Dyck lattices. In particular, in the case of Dyck and Schröder lattices, we obtain a combinatorial interpretation for the Euler characteristic in terms of the number of tunnels of a path [10,11]. The case of Motzkin lattices cannot be dealt with using the machinery developed for Dyck-like lattices. So, we provide an ad hoc argument which allows to obtain a (not obvious) combinatorial interpretation of the Euler characteristic also for these lattices.

\section{Basic definitions and properties}

As usual, $\mathbb{N}$ denotes the set of all non-negative integers, $\mathbb{Z}$ denotes the set of all integers, $[n]=\{1,2, \ldots, n\}$ and $[0, n]=\{0,1, \ldots, n\}$. For any real number $x,\lfloor x\rfloor$ is the greatest integer less than or equal to $x$, whereas $\lceil x\rceil$ is the least integer greater than or equal to $x$. In the rest of this section, we will recall some basic definitions concerning lattice paths and the theory of partially ordered sets $[1,5,8]$.

\subsection{Lattice paths}

Given a finite subset $\Gamma \subseteq \mathbb{Z}$, a $\Gamma$-path of length $n$ is a function $f:[0, n] \rightarrow \mathbb{N}$ such that $f(0)=f(n)=0$ and $f(k+1)-f(k) \in \Gamma$, for every $k \in[0, n-1]$. Equivalently, a $\Gamma$-path of length $n$ is a sequence of $n$ steps $(1, k)$, with $k \in \Gamma$, starting from the origin, ending on the $x$-axis and never going below that axis.

Considered as functions, $\Gamma$-paths of length $n$ can be ordered coordinatewise setting $f \leq g$ whenever $f(k) \leq g(k)$ for every $k \in[0, n]$. In this way, we obtain a poset $\mathcal{C}_{n}^{\Gamma}$ which, under suitable conditions [13], turns out to be a lattice. In particular, $\mathcal{C}_{n}^{\Gamma}$ is a distributive lattice when $\Gamma=\{-b, a\}$, with 
$a, b \in \mathbb{N}$. Here $\{-b, a\}$-paths will be called Dyck-like paths of type $(a, b)$, since they are a natural generalization of ordinary Dyck paths (corresponding to the case $a=b=1$ ). Dyck-like paths have already been considered in [9], where several results are proved and the case $(a, b)=(3,2)$ is examined in great detail, and also in [2], where they are used as a source of examples.

We will use $U, H$ and $D$ to denote up steps, horizontal steps and down steps. Given a path $\gamma$ having precisely one type of up step and one type of down step, a peak of $\gamma$ is just a sequence formed by an up step and a down step. An elevated Dyck path is a Dyck path touching the $x$-axis just at its starting and ending points.

In the sequel, a path will be denoted by a Latin letter when considered as an element of a lattice and by a Greek letter in all other cases.

\subsection{Partially ordered sets}

In a poset $P$, an element $x$ is covered by an element $y$ when $x \leq u \leq y$ implies $x=u$ or $u=y$. A poset $P$ is ranked when it admits a rank function, that is a function $r: P \rightarrow \mathbb{N}$ such that $r(y)=r(x)+1$ whenever $x$ is covered by $y$. For finite posets the function $r$ is usually chosen so that the minimal elements have rank 0 . The height of $P$ is its maximum rank.

A map $f: P \rightarrow Q$ between two posets $P$ and $Q$ is order-preserving when $x \leq y$ implies $f(x) \leq f(y)$ for every $x, y \in P$. In particular, it is a poset isomorphism when it is an order-preserving bijection, whose inverse is order-preserving, too. In this paper, all isomorphisms between posets are always understood to be poset isomorphisms.

A join-semilattice (meet-semilattice) is a poset in which there exists the supremum (the infimum) of any two elements. A lattice is a poset in which there exists the supremum and the infimum of any two elements.

A poset $P$ has a minimum $\widehat{0}$ (maximum $\widehat{1}$ ) when it has only one minimal (maximal) element. In a poset $P$ with minimum (maximum), an atom (coatom) is an element covering the minimum (covered by the maximum). In a (finite) lattice $L$, the socle is the join of all atoms and the radical is the meet of all coatoms.

An order-ideal of a poset $P$ is a subset $I$ such that $x \in I$ and $u \leq x$ imply $u \in I$. The principal ideal $\downarrow x$ generated by an element $x \in P$ is the set of all elements $u \in P$ such that $u \leq x$. Similarly, the principal filter $\uparrow x$ generated by an element $x \in P$ is the set of all elements $u \in P$ such that $u \geq x$.

A join-irreducible element of a distributive lattice $\mathcal{D}$ is any element $x \neq \widehat{0}$ with the property that if $x=u \vee v$ then $x=u$ or $x=v$. The set $\mathcal{J}(P)$ of all order-ideals of $P$, ordered by inclusion, is a distributive lattice. Conversely, 
by Birkhoff's representation theorem, every finite distributive lattice $\mathcal{D}$ is isomorphic to the lattice $\mathcal{J}(P)$ where $P=\operatorname{Spec}(\mathcal{D})$, the spectrum of $\mathcal{D}$, is defined as the poset of all join-irreducibles of $\mathcal{D}$.

A valuation on a distributive lattice $\mathcal{D}$ with values in $\mathbb{R}$ is a function $\nu: \mathcal{D} \rightarrow \mathbb{R}$ such that $\nu(\widehat{0})=0$ and $\nu(x \vee y)+\nu(x \wedge y)=\nu(x)+\nu(y)$ for every $x, y \in \mathcal{D}$. A valuation on a finite distributive lattice $\mathcal{D}$ is uniquely determined by the values it takes on the set of join-irreducibles of $\mathcal{D}$, and these values can be arbitrarily assigned [18]. Every valuation $\nu$ satisfies the following generalized form of the principle of inclusion-exclusion:

$$
\nu\left(x_{1} \vee \cdots \vee x_{n}\right)=\sum_{\substack{S \subseteq[n] \\ S \neq \emptyset}}(-1)^{|S|-1} \nu\left(\bigwedge_{i \in S} x_{i}\right) .
$$

The $($ Euler $)$ characteristic of $\mathcal{D}$ is defined as the unique valuation $\chi$ such that $\chi(\widehat{0})=0$ and $\chi(x)=1$ for every join-irreducible $x$ of $\mathcal{D}$. In particular, $\chi(D)=\chi(\widehat{1})$.

A partition of a non-negative integer $n$ is a sequence $\lambda=\left(\lambda_{1}, \lambda_{2}, \ldots, \lambda_{k}\right)$ of positive integers such that $\lambda_{1} \geq \lambda_{2} \geq \cdots \geq \lambda_{k}>0$ and $\lambda_{1}+\lambda_{2}+\cdots+\lambda_{k}=$ $n$. The $\lambda_{i}$ 's are called the parts of $\lambda$, and the sum of all parts of $\lambda$ will be denoted by $|\lambda|$. The (Ferrers) diagram of $\lambda$ is a left-justified array of squares (or dots) with exactly $\lambda_{i}$ squares in the $i$-th row. Partitions can be ordered by magnitude of parts [1]: if $\alpha=\left(a_{1}, \ldots, a_{h}\right)$ and $\beta=\left(b_{1}, \ldots, b_{k}\right)$, then $\alpha \leq \beta$ whenever $h \leq k$ and $a_{i} \leq b_{i}$ for every $i=1,2, \ldots, h$. If $\alpha \leq \beta$ the diagram of $\alpha$ is contained in the diagram of $\beta$. The resulting poset is an infinite distributive lattice, called Young lattice. In particular, the Young lattice $\mathcal{Y}_{\lambda}$ generated by a partition $\lambda$ is the set of all integer partitions $\alpha$ such that $\alpha \leq \lambda$, i.e. $\mathcal{Y}_{\lambda}=\downarrow \lambda$ in $\mathcal{Y}$.

\section{Dyck-like paths}

\subsection{Representation of Dyck-like paths}

To study Dyck-like paths of type $(a, b)$ we can always suppose, without loss of generality, that $a \geq b$. We begin our study noticing that the length of a Dyck-like path of type $(a, b)$ strictly depends on $a$ and $b$, as stated in the following proposition essentially due to Duchon [9].

Proposition 3.1. Every Dyck-like path of type $(a, b)$ has $\frac{b}{\operatorname{gcd}(a, b)} \cdot n$ up steps and $\frac{a}{\operatorname{gcd}(a, b)} \cdot n$ down steps, for some $n \in \mathbb{N}^{*}$, and it ends at the point $(n$. $\ell(a, b), 0)$, where $\ell(a, b)=\frac{a+b}{\operatorname{gcd}(a, b)}$. 


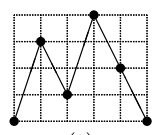

(i)

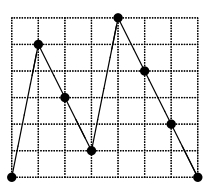

(ii)

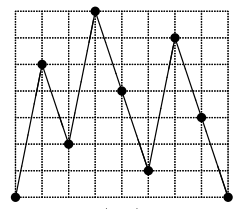

(iii)

Figure 1: The minimum element in $\mathcal{D}_{1}^{(a, b)}$ for (i) $a=3, b=2$, (ii) $a=5$, $b=2$ and (iii) $a=5, b=3$.

Proof. Let $h, k$ be the number of up steps and down steps of the path respectively. Then, $h a=k b$. It follows that $h a_{1}=k b_{1}$, where $a_{1}=\frac{a}{\operatorname{gcd}(a, b)}$ and $b_{1}=\frac{b}{\operatorname{gcd}(a, b)}$. Clearly, since $\operatorname{gcd}\left(a_{1}, b_{1}\right)=1$, it follows that there exists $n \in \mathbb{N}^{*}$ such that $h=n b_{1}$ and $k=n a_{1}$. Thus, the path contains $n \cdot \ell(a, b)$ steps, so that it ends at the point $(n \cdot \ell(a, b), 0)$.

The lattice of all Dyck-like paths of type $(a, b)$ having length $n \cdot \ell(a, b)$ will be denoted by $\mathcal{D}_{n}^{(a, b)}$.

Set $h=\frac{b}{\operatorname{gcd}(a, b)} \cdot n, k=\frac{a}{\operatorname{gcd}(a, b)} \cdot n$ and $r_{1}+r_{2}+\cdots r_{i}=\left\lfloor\frac{i a}{b}\right\rfloor$, for every $i \in[h] . \mathcal{D}_{n}^{(a, b)}$ is a distributive lattice of height $\left(\begin{array}{c}h \\ 2\end{array}\right)$, with minimum $\widehat{0}=U D^{r_{1}} U D^{r_{2}} \cdots U D^{r_{h}}$ (see Figure 1 for some examples) and maximum $\widehat{1}=$ $U^{h} D^{k}$, with $h-1$ atoms of the form $U D^{r_{1}} U D^{r_{2}} \cdots U D^{r_{i}-1} U D^{r_{i+1}+1} \cdots U D^{r_{h}}$, and just 1 coatom $U^{h-1} D U D^{k-1}$. It is easy to see that the socle of $\mathcal{D}_{n}^{(a, b)}$ is the path $s=U D^{r_{1}-1} U D^{r_{2}} \cdots U D^{r_{h-1}} U D^{r_{h}+1}$ and that the principal filter $\uparrow s$ is isomorphic to $\mathcal{D}_{h-1}^{(a, b)}$, whenever $n \geq 1$. The special (and important) case of ordinary Dyck lattices $\mathcal{D}_{n}$ corresponds to $(a, b)=(1,1)$, and for them we can also observe that the principal ideal $\downarrow s$ is isomorphic to the Boolean algebra $B_{n-1}$ having $n-1$ atoms. For Dyck lattices, see also Figure 3.

Our next goal is to find some suitable representation results for the lattices $\mathcal{D}_{n}^{(a, b)}$. We begin by giving an alternative description of $\mathcal{D}_{n}^{(a, b)}$. For each path in $\mathcal{D}_{n}^{(a, b)}$, consider the path obtained by replacing each step $(1, a)$ with a step $(1,1)$ and each step $(1,-b)$ with a step $(1,-1)$. If $a \neq b$, the paths of the resulting set $\overline{\mathcal{D}}_{n}^{(a, b)}$ terminates below the $x$-axis.

Lemma 3.2. The lattice $\overline{\mathcal{D}}_{n}^{(a, b)}$ consists of all the paths that start from the origin, end at the point $\left(n \cdot \frac{a+b}{\operatorname{gcd}(a, b)}, n \cdot \frac{b-a}{\operatorname{gcd}(a, b)}\right)$ and never go below the line $y=\frac{b-a}{a+b} x$. 


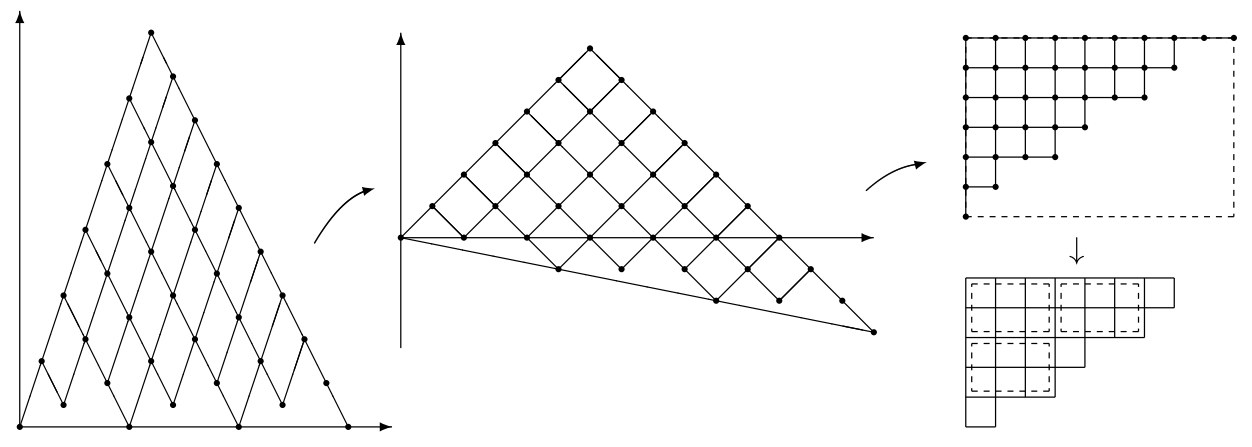

Figure 2: A path in $\mathcal{D}_{n}^{(a, b)}$ and the associated partition for $a=3, b=2$ and $n=3$.
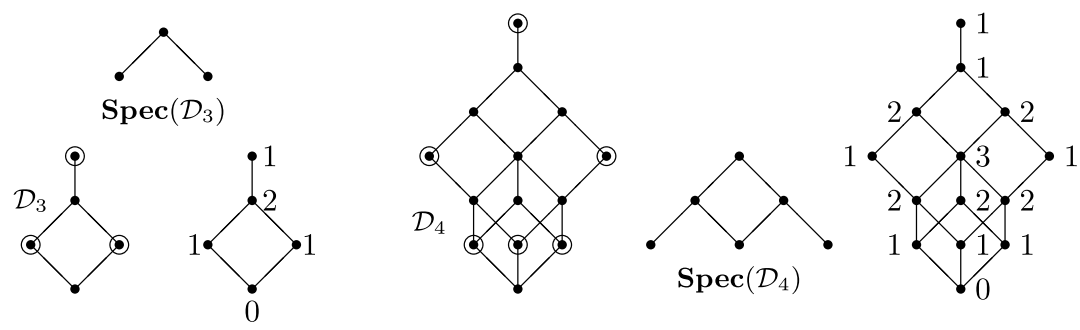

Figure 3: The Dyck lattices $\mathcal{D}_{3}$ and $\mathcal{D}_{4}$, their spectra and the distribution of the characteristic.

Proof. If $\left(x_{0}, y_{0}\right)$ is the endpoint of the paths in $\overline{\mathcal{D}}_{n}^{(a, b)}$ then $x_{0}$ is equal to the length of the paths in $\mathcal{D}_{n}^{(a, b)}$ obtained in Proposition 3.1, and $y_{0}$ can be obtained by subtracting from the number $h$ of steps $(1,1)$ the number $k$ of steps $(1,-1)$. Since $h$ and $k$ are defined as in Proposition 3.1, it follows that $y_{0}=n \cdot \frac{b-a}{\operatorname{gcd}(a, b)}$. The last part of the thesis is obtained by determining the line passing through the origin and the point $\left(x_{0}, y_{0}\right)$.

A careful inspection of some examples (as the one in Figure 2) suggests that the paths in $\overline{\mathcal{D}}_{n}^{(a, b)}$ (or, equivalently, in $\mathcal{D}_{n}^{(a, b)}$ ) are in bijection with the integer partitions whose Ferrers diagram is included in the Ferrers diagram obtained by taking simultaneously the minimum and the maximum paths in $\overline{\mathcal{D}}_{n}^{(a, b)}$, then rotating by $45^{\circ}$ anticlockwise and finally considering the squares obtained by drawing the part of the lattice grid included between the two paths. We will denote such a partition $\lambda_{n}^{(a, b)}$, and call it the partition associated with $\overline{\mathcal{D}}_{n}^{(a, b)}\left(\right.$ or $\left.\mathcal{D}_{n}^{(a, b)}\right)$. 
All this implies

Proposition 3.3. The distributive lattice $\mathcal{D}_{n}^{(a, b)}$ is isomorphic to the dual of the Young lattice $\mathcal{Y}_{\lambda_{n}^{(a, b)}}$.

Next proposition describes the partition $\lambda_{n}^{(a, b)}$ in terms of the positive integers $a, b$ and $n$, when $\operatorname{gcd}(a, b)=1$ (the general case follows immediately).

Proposition 3.4. Let $\operatorname{gcd}(a, b)=1$. For any $n \in \mathbb{N}$,

$$
\lambda_{n}^{(a, b)}=\left(\lambda_{n, b}, \ldots, \lambda_{n, 1}, \lambda_{n-1, b}, \ldots, \lambda_{n-1,1}, \ldots, \lambda_{2, b}, \ldots, \lambda_{2,1}, \lambda_{1, b}, \ldots, \lambda_{1,2}\right)
$$

is a partition with $n b-1$ parts, where $\lambda_{h, k}=(h-1) a+\lfloor(k-1) \cdot a / b\rfloor$. In particular,

$$
\left|\lambda_{n}^{(a, b)}\right|=a b \frac{n(n-1)}{2}+n\left|\lambda_{1}^{(a, b)}\right|
$$

Proof. From the expression of $\widehat{0}$ it follows that the $i$-th row of $\lambda_{n}^{(a, b)}$ (counting upwards) contains $r_{1}+r_{2}+\cdots+r_{i}=\left\lfloor\frac{i a}{b}\right\rfloor$ squares, for every $i \in[n b-1]$. Since $\lambda_{h, k}$ is the number of squares which are contained in the $((h-1) b+(k-1))$-th row of $\lambda_{n}^{(a, b)}$, where $(h, k) \in[\lfloor a\rfloor \times\lfloor b\rfloor] \backslash\{(1,1)\}$, we obtain that

$$
\lambda_{h, k}=\left\lfloor\frac{(h-1) b+(k-1)}{b} a\right\rfloor=h-1+\left\lfloor\frac{(k-1) a}{b}\right\rfloor .
$$

Since Dyck paths are Dyck-like with $(a, b)=(1,1)$, the following corollary holds.

Corollary 3.5. The Dyck lattice $\mathcal{D}_{n}$ is isomorphic to the dual of the Young lattice $\mathcal{Y}_{\lambda_{n}}$, where $\lambda_{n}=(n-1, n-2, \ldots, 2,1)$.

Before proceeding further, it will be useful to characterize the joinirreducible elements of $\mathcal{D}_{n}^{(a, b)}$.

Proposition 3.6. A path in $\mathcal{D}_{n}^{(a, b)}$ (resp. $\overline{\mathcal{D}}_{n}^{(a, b)}$ ) is join-irreducible if and only if it contains exactly one peak (weakly) above the line $y=a+b$ (resp. $y=$ $\left.\frac{b-a}{a+b} x+2\right)$. Furthermore, this peak determines the path uniquely.

Proof. In $\mathcal{D}_{n}^{(a, b)}$ a path $x$ is covered by a path $y$ if it can be obtained from $y$ by changing a peak $U D$ into a valley $D U$. The paths with a unique peak (weakly) above the line $y=a+b$ are the only paths for which this operation can be performed just in one way. This argument can be trivially adapted to obtain the corresponding statement for $\overline{\mathcal{D}}_{n}^{(a, b)}$. 
An easy corollary concerning Dyck paths is the following.

Corollary 3.7. The join-irreducibles of the lattice $\mathcal{D}_{n}$ are the paths with exactly one peak (weakly) above the line $y=2$, that is the paths of the form

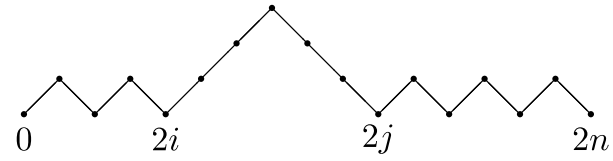

In particular, the spectrum of $\mathcal{D}_{n}$ is isomorphic to the poset of the intervals of a chain $\mathcal{C}_{n-2}$ with $n-1$ elements, i.e. $\operatorname{Spec}\left(\mathcal{D}_{n}\right) \simeq\left\{(i, j) \in \mathcal{C}_{n}^{2}: i \leq\right.$ $j-2\} \simeq \operatorname{Int}\left(\mathcal{C}_{n-2}\right)$.

Proof. The last statement can be proved by simply observing that every join-irreducible in $\mathcal{D}_{n}$ is uniquely determined by the interval corresponding to its unique peak (weakly) above the line $y=2$.

Now, we can state our main result concerning the representation of Dycklike paths.

Theorem 3.8. The distributive lattice $\mathcal{D}_{n}^{(a, b)}$ is isomorphic to $\mathcal{J}\left(P_{n}^{(a, b)}\right)$, where $P_{n}^{(a, b)}$ is the set of points $(x, y) \in[0, n \cdot \ell(a, b)] \times \mathbb{Z}$ such that $x$ and $y$ have the same parity, $y \leq x, y \leq-x+n \frac{2 b}{\operatorname{gcd}(a, b)}$ and $y \geq \frac{b-a}{a+b}+2$, ordered coordinatewise.

Proof. This follows from Proposition 3.3, by noticing that $P_{n}^{(a, b)}$ coincides with the set of the peaks weakly above the line $y \geq \frac{b-a}{a+b}+2$ of the joinirreducibles in $\overline{\mathcal{D}}_{n}^{(a, b)}$.

The rank of a path $x \in \mathcal{D}_{n}^{(a, b)}$ can be easily expressed in terms of its area $\mathcal{A}(x)$, i.e. the area of the region determined by the path and the $x$-axis.

Proposition 3.9. The rank of any element $x \in \mathcal{D}_{n}^{(a, b)}$ is

$$
r(x)=\frac{\mathcal{A}(x)-\mathcal{A}(\widehat{0})}{a+b} .
$$

Proof. We will prove that the function defined in (2) satisfies the properties of a rank function. First we have $r(\widehat{0})=\frac{\mathcal{A}(\widehat{0})-\mathcal{A}(\widehat{0})}{a+b}=0$. Suppose now that $x$ is covered by $y$. Then the path $y$ is obtained from $x$ by replacing a valley with a peak. This implies that the area $A(y)$ is obtained from $\mathcal{A}(x)$ by adding the area of a parallelogram of area $a+b$. Hence $r(y)=\frac{\mathcal{A}(x)+a+b-\mathcal{A}(\widehat{0})}{a+b}=$ $r(x)+1$.

In the special case of Dyck lattices, the above formula reads $r(x)=$ $(\mathcal{A}(x)-n) / 2$. 


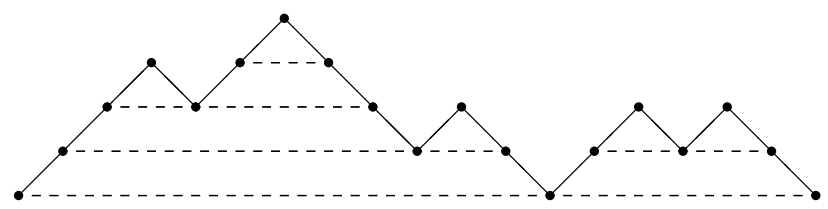

Figure 4: A Dyck path $\gamma$ of length 18 with its 9 tunnels (two 0-tunnels, four 1-tunnels, two 2-tunnels and one 3-tunnel).

Before closing the present section, we mention some important facts concerning Dyck lattices which will be fundamental in what follows.

A pyramid in a Dyck path is a maximal sequence of consecutive steps of the form $U^{h} D^{h}$, for some $h \geq 1$, which can be replaced with $U^{h-1} D U D^{h-1}$ still remaining inside the class of Dyck paths. The positive integer $h$ is called the dimension of the pyramid, whereas the height of the vertex is called the height of the pyramid.

A $k$-tunnel of a Dyck path $x$ is any segment, not reducing to a point, on the horizontal line $y=k$ having in common with $x$ only its extreme points $[10,11]$ (see Figure 4 for an example). Clearly every tunnel corresponds to a factor of the form $U y D$ where the steps $U$ and $D$ are at the same level (see again Figure 4). In particular, the 0-tunnels correspond to the primitive factors of the paths.

Proposition 3.10. The meet of two join-irreducibles of $\mathcal{D}_{n}$ is $\widehat{0}$ or a joinirreducible, i.e. $\{\widehat{0}\} \cup \operatorname{Spec}\left(\mathcal{D}_{n}\right)$ is a ranked sub-meet-semilattice of $\mathcal{D}_{n}$. Moreover, the rank of a path in the poset $\{\widehat{0}\} \cup \operatorname{Spec}\left(\mathcal{D}_{n}\right)$ is equal to the maximum height of its tunnels (i.e. $r(x)=k$ whenever $x$ has a $k$-tunnel but not a $(k+1)$-tunnel).

Proof. Two join-irreducible Dyck paths either meet in $\widehat{0}$ or have pyramids intersecting in a single point with integer coordinates.

We observe that an analogous result also holds for the lattices $\mathcal{D}_{n}^{(a, b)}$, i.e. it can be shown that $\{\widehat{0}\} \cup \operatorname{Spec}\left(\mathcal{D}_{n}^{(a, b)}\right)$ is a sub-meet-semilattice of $\mathcal{D}_{n}^{(a, b)}$, even if it is in general not ranked.

\subsection{Characteristic}

A Dyck-like lattice is a distributive lattice whose spectrum is a ranked poset admitting a labelling of its elements with the following properties: all labels are positive integers and every antichain $S=\left\{s_{1}, \ldots, s_{n}\right\}$ of join-irreducibles can be linearly ordered so that the labels of the elements of $S$ are distinct and, if $s_{1}$ and $s_{n}$ are the elements having minimum and maximum labels, 
respectively, then $s_{1} \wedge s_{n}=s_{1} \wedge s_{2} \wedge \cdots \wedge s_{n-1} \wedge s_{n}$. In a Dyck-like lattice, any labelling of the join-irreducibles satisfying the above properties will be called a Dyck-like labelling. Similarly, if $\left\{s_{1}, \ldots, s_{n}\right\}$ is an antichain of joinirreducibles as above, the ordered $n$-tuple $\left(s_{1}, \ldots, s_{n}\right)$ will be called a Dycklike antichain.

Proposition 3.11. For any $a, b \in \mathbb{N}$ and for every $n \in \mathbb{N}$, the lattice $\mathcal{D}_{n}^{(a, b)}$ is a Dyck-like lattice.

Proof. Consider the lattice $\overline{\mathcal{D}}_{n}^{(a, b)}$ isomorphic to $\mathcal{D}_{n}^{(a, b)}$, as defined in Section 3.1. Label each join-irreducible with the abscissa of its highest peak. Such a labelling is a Dyck-like labelling. The fact that the spectrum of $\overline{\mathcal{D}}_{n}^{(a, b)}$ is ranked is a consequence of Theorem 3.8.

We conjecture that a sort of converse of the previous proposition holds. More precisely, the following assertion seems plausible:

Conjecture 3.12. Every finite Dyck-like lattice can be represented as a sublattice of a lattice of Dyck-like paths of suitable length.

We will say that an element $x$ of a distributive lattice $\mathcal{D}$ is quasi-joinirreducible when there exists an ordered $k$-tuple $\left(s_{1}, \ldots, s_{k}\right)$ forming an antichain of join-irreducibles such that $x=s_{1} \vee \cdots \vee s_{k}$ and $s_{i} \wedge s_{i+1} \neq \widehat{0}$, for every $i=1,2, \ldots, k-1$.

Lemma 3.13. Let $\mathcal{D}$ be a Dyck-like lattice and $x \in \mathcal{D}$ a quasi-join-irreducible. Then $x$ can be expressed as $x=t_{1} \vee \cdots \vee t_{k}$ where $\left(t_{1}, \ldots, t_{k}\right)$ is a Dyck-like antichain and $t_{i} \wedge t_{i+1} \neq \hat{0}$, for every $i=1,2, \ldots, k-1$.

Proof. Let $x=t_{1} \vee \cdots \vee t_{k}$, where $\left(t_{1}, \ldots, t_{k}\right)$ is a Dyck-like antichain. Suppose there exists an index $j<k$ such that $t_{j} \wedge t_{j+1}=\hat{0}$. This would imply $t_{a} \wedge t_{b}=\hat{0}$, for any $a \leq j$ and $b \geq j+1$. However, in any rearrangement of the $t_{i}$ 's, at least a pair of adjacent elements must appear such that one has index $\leq j$ and the other has index $\geq j+1$, and this contradicts the hypothesis that $x$ is quasi-join-irreducible.

Lemma 3.13 asserts that in a Dyck-like lattice the antichain of joinirreducibles in the definition of a quasi-join-irreducible element can be taken to be a Dyck-like antichain.

Before proceeding further we notice that, in the finite case, the notion of a quasi-join-irreducible element can be related to an interesting property.

Proposition 3.14. Suppose that $\mathcal{D}$ is a finite distributive lattice. If $x \in \mathcal{D}$ is quasi-join-irreducible then the following property holds:

$$
\text { if } x=y \vee z \text { and } y \wedge z=\widehat{0} \text {, then } x=y \text { or } x=z \text {. }
$$


Proof. In a finite distributive lattice, every element has an essentially unique factorization (up to the order of the factors) as a join of join-irreducible elements. Let $x=y \vee z$ and $y \wedge z=\widehat{0}$. Let $x=s_{1} \vee \cdots \vee s_{k}$, with $s_{1}, \ldots, s_{k}$ join-irreducibles as in the definition of a quasi-join-irreducible. Then necessarily both $y$ and $z$ can be expressed as the join of some of the $s_{i}$ 's. Moreover, there cannot exist any $i$ such that $s_{i}$ appears in the factorization of both $y$ and $z$, otherwise $y \wedge z \neq \widehat{0}$. Therefore the set $\left\{s_{1}, \ldots s_{k}\right\}$ can be partitioned into two blocks (the elements appearing in the factorization of $y$ and those appearing in the factorization of $z$, respectively) in such a way that, if $s_{i}$ and $s_{j}$ belong to different blocks, then $s_{i} \wedge s_{j}=\widehat{0}$. The fact that $x$ is quasijoin-irreducible implies that one of the two blocks must be empty, whence $x=y$ or $x=z$.

We can also prove a converse of the above proposition in the hypothesis that the lattice is Dyck-like.

Proposition 3.15. Suppose that $\mathcal{D}$ is a finite Dyck-like lattice. If $x \in \mathcal{D}$ satisfies property (3) (for all $y$ and $z$ ), then it is quasi-join-irreducible.

Proof. Set $x=s_{1} \vee \cdots \vee s_{k}$, where the $s_{i}$ 's are join-irreducibles and $\left(s_{1}, \ldots, s_{k}\right)$ is a Dyck-like antichain. If $x$ were not quasi-join-irreducible, then there would exist $i$ such that $s_{i} \wedge s_{i+1}=\widehat{0}$. Define $y=s_{1} \vee \cdots \vee s_{i}$ and $z=s_{i+1} \vee \ldots \vee s_{k}$. Of course $x=y \vee z$. Moreover, for every $a \leq i$ and for every $b \geq i+1$, $s_{a} \wedge s_{b}=s_{a} \wedge \cdots \wedge s_{i} \wedge s_{i+1} \wedge \cdots \wedge s_{b}=\widehat{0}$, whence $y \wedge z=\widehat{0}$. Therefore $x=y$ or $x=z$, which is impossible since $x$ has a unique factorization as the join of join-irreducibles.

Remark. In the above proofs, the finiteness hypothesis is used in order to ensure the uniqueness of the factorization as join of join-irreducibles. Actually, such a property is guaranteed also if we relax the hypothesis, and simply ask that the distributive lattice $\mathcal{D}$ satisfies the descending chain condition, i.e. every nonempty subset of $\mathcal{D}$ has minimal elements (see, for instance, $[7])$. So the above propositions can be reformulated in this more general setting.

A special Dyck-like lattice is a Dyck-like lattice where the meet of any two join-irreducibles is $\widehat{0}$ or a join-irreducible. In view of Proposition 3.10 and Proposition 3.11, it follows that $\mathcal{D}_{n}^{(a, b)}$ is a special Dyck-like lattice.

Proposition 3.16. In a special Dyck-like lattice $\mathcal{D}$, every quasi-join-irreducible element has Euler characteristic equal to 1.

Proof. Let $x$ be a quasi-join-irreducible element of $\mathcal{D}$. Then $x=s_{1} \vee \cdots \vee s_{k}$, where $s_{1}, \ldots, s_{k}$ are incomparable join-irreducibles such that $s_{i} \wedge s_{i+1} \neq \widehat{0}$ for every $i<k$. If $k=1$ then $x$ is join-irreducible and $\chi(x)=1$. From 
formula (1) we have

$$
\chi(x)=\chi\left(s_{1} \vee \cdots \vee s_{k}\right)=\sum_{\substack{S \subseteq[k] \\ S \neq \emptyset}}(-1)^{|S|-1} \chi\left(\bigwedge_{i \in S} s_{i}\right) .
$$

By Lemma 3.13, $\left(s_{1}, \ldots, s_{k}\right)$ can be taken to be a Dyck-like antichain. Hence it follows at once that $\bigwedge_{i \in S} s_{i}=s_{i_{1}} \wedge s_{i_{2}}$, where $i_{1}=\min S$ and $i_{2}=\max S$. If $S$ is a subset of $[k]$ with $\min S=\max S$, then clearly $|S|=1$. Hence the contribution of these subsets to the sum in (4) is $\chi\left(s_{1}\right)+\cdots+\chi\left(s_{k}\right)=k$. If $S$ is a subset of $[k]$ with $\max S=\min S+1$, then it follows that $S=\{i, i+1\}$. Since $s_{i} \wedge s_{i+1}$ is a join-irreducible, the contribution of these subsets to the sum is

$$
-\sum_{i=1}^{k-1} \chi\left(s_{i} \wedge s_{i+1}\right)=-(k-1)
$$

Finally, since the subsets $S$ of $[k]$ with $\max S-\min S=j \geq 2$, having minimum $i$, maximum $i+j$ and cardinality $h+2(h \geq 0)$ are exactly $\left(\begin{array}{c}j-1 \\ h\end{array}\right)$, the contribution of all these subsets is

$$
\sum_{h=0}^{j-1}\left(\begin{array}{c}
j-1 \\
h
\end{array}\right)(-1)^{h+1} \chi\left(s_{i} \wedge s_{i+j}\right)=-(1-1)^{j-1} \chi\left(s_{i} \wedge s_{i+j}\right)=0 .
$$

In conclusion, we have $\chi(x)=k-(k-1)=1$.

An element $x$ of a finite distributive lattice is said to have a quasi-joinirreducible decomposition when it can be expressed as a join of quasi-joinirreducible elements $x_{1}, \ldots, x_{k}$ such that $x_{i} \wedge x_{j}=\widehat{0}$, for every $i \neq j$.

Proposition 3.17. Every element $x \neq \widehat{0}$ of a finite Dyck-like lattice has a quasi-join-irreducible decomposition.

Proof. Let $x=s_{1} \vee \cdots \vee s_{k}$, where $\left(s_{1}, \ldots, s_{k}\right)$ is a Dyck-like antichain. If $i$ is the first index such that $s_{i} \wedge s_{i+1}=\widehat{0}$ then $s_{1} \wedge \cdots \wedge s_{i}$ is quasi-joinirreducible. If $j$ is the second index satisfying the above condition (and so $\left.s_{j} \wedge s_{j+1}=\widehat{0}\right)$, then obviously $s_{i+1} \wedge \cdots \wedge s_{j}$ is quasi-join-irreducible and $\left(s_{1} \wedge \cdots \wedge s_{i}\right) \wedge\left(s_{i+1} \wedge \cdots \wedge s_{j}\right)=\widehat{0}$. Repeating this argument one obtains the desired decomposition.

We observe that the uniqueness of the quasi-join-irreducible decomposition can be proved for a generic finite distributive lattice.

Lemma 3.18. Let $x$ be a quasi-join-irreducible of a finite distributive lattice. If $x \leq y \vee z$ and $y \wedge z=\widehat{0}$, then $x \leq y$ or $x \leq z$. 
Proof. From the hypothesis we get $x=x \wedge(y \vee z)=(x \wedge y) \vee(x \wedge z)$. Moreover, from $y \wedge z=\widehat{0}$, it follows that $(x \wedge y) \wedge(x \wedge z)=\widehat{0}$. Since $x$ is quasi-join-irreducible, Proposition 3.14 implies that $x=x \wedge y$ or $x=x \wedge y$, that is $x \leq y$ or $x \leq y$.

Proposition 3.19. In a finite distributive lattice, the quasi-join-irreducible decomposition of an element is unique (up to the order of factors).

Proof. Let $x=x_{1} \vee \cdots \vee x_{s}=y_{1} \vee \cdots \vee y_{r}$ be two quasi-join-irreducible decompositions of $x$. For every $i \leq s$, the above lemma implies that there exists $j \leq r$ such that $x_{i} \leq y_{j}$ and, analogously, that there exists $k \leq s$ such that $y_{j} \leq x_{k}$. Therefore $x_{i} \leq x_{k}$, whence $x_{i}=x_{k}$, and so also $x_{i}=$ $y_{j}$. We have thus shown that $\left\{x_{1}, \ldots, x_{s}\right\} \subseteq\left\{y_{1}, \ldots, y_{t}\right\}$, and the opposite inequality holds for a similar reason.

Theorem 3.20. Let $\mathcal{D}$ be a finite special Dyck-like lattice. Then, for every $x \in \mathcal{D} \backslash\{\widehat{0}\}, \chi(x)$ is the number of quasi-join-irreducibles in a decomposition of $x$.

Proof. If $x=x_{1} \vee \cdots \vee x_{k}$ is a quasi-join-irreducible decomposition of $x$, then it follows at once that $\chi(x)=\chi\left(x_{1}\right)+\cdots+\chi\left(x_{k}\right)=k$.

In the case of Dyck paths, the characteristic can be interpreted combinatorially as follows.

Proposition 3.21. A Dyck path $x \in \mathcal{D}_{n}$ is quasi-join-irreducible if and only if it has precisely one 1-tunnel.

Proof. If $x$ has precisely one 1-tunnel, then it is of the form $(U D)^{s} U y D(U D)^{t}$, for suitable $s, t \in \mathbb{N}$, where $y$ is an elevated Dyck path. Then $x$ can be expressed as $x=x_{1} \vee \cdots \vee x_{k}$, where $x_{1}, \ldots, x_{k}$ are the join-irreducibles uniquely determined by the peaks of $y$. The fact that $y$ is elevated implies that $x_{i} \wedge x_{i+1} \neq \widehat{0}$, for every $i<k$, and consequently that $x$ is quasi-joinirreducible.

On the other hand, suppose that $x \in \mathcal{D}_{n}$ is quasi-join-irreducible. If $x$ had no 1 -tunnels, then $x=\widehat{0}$, which is impossible (since $\widehat{0}$ is not quasijoin-irreducible). If $x$ had more than one 1 -tunnel, then $x$ would have at least two elevated factors (i.e. two Dyck factors starting at level 1). In this situation, any expression of $x$ as a join of join-irreducibles would contain join-irreducible elements determined by the peaks of all the elevated factors of $x$. Thus, if $x=x_{1} \vee \cdots \vee x_{k}$ is any join-irreducible decomposition of $x$, then there exists at least one $i<k$ such that $x_{i}$ and $x_{i+1}$ are joinirreducibles determined by the peaks of two different elevated factors, and so $x_{i} \wedge x_{i+1}=\widehat{0}$. In conclusion, $x$ has exactly one 1 -tunnel. 
Finally, as an immediate consequence of Theorem 3.20 and Proposition 3.21 , we have

Theorem 3.22. The characteristic of a Dyck path is the number of its 1-tunnels.

\subsection{Generalized characteristics}

Suppose that $\mathcal{D}$ is a finite special Dyck-like lattice with spectrum $P$ such that $\widehat{P}=\{\widehat{0}\} \cup P$ is ranked with rank function $r_{\widehat{P}}$. For every $k \in \mathbb{N}^{*}$, we define the generalized characteristic $\chi_{k}$ as the valuation on $\mathcal{D}$ such that

$$
\chi_{k}(x)= \begin{cases}1 & \text { if } r_{\widehat{P}}(x) \geq k \\ 0 & \text { if } r_{\widehat{P}}(x)<k\end{cases}
$$

for every join-irreducible $x$. Clearly $\chi_{1}$ is equal to the ordinary characteristic $\chi$. Our aim is to evaluate $\chi_{k}(x)$ for every $x \in \mathcal{D}$.

Proposition 3.23. Let $x=x_{1} \vee \cdots \vee x_{m}$ where $m \geq 1$, each $x_{i}$ is a joinirreducible and $r_{\widehat{P}}\left(x_{j} \wedge x_{j+1}\right) \geq k$ for every $j<m$. Then $\chi_{k}(x)=1$.

Proof. The proof follows the same lines of that of Proposition 3.16.

Proposition 3.24. Let $x=x_{1} \vee \cdots \vee x_{m}$, where $m \geq 1$, and each $x_{i}=$ $x_{i, 1} \vee \cdots \vee x_{i, \ell_{i}}$ is a join of join-irreducibles such that $r_{\widehat{P}}\left(x_{i, t} \wedge x_{i, t+1}\right) \geq k$, for every $t=1, \ldots, \ell_{i}-1$, and $r_{\widehat{P}}\left(x_{i, t} \wedge x_{j, s}\right)<k$ for $t=1, \ldots, \ell_{i}, s=1, \ldots, \ell_{j}$, $i \neq j$. Then $\chi_{k}(x)=m$.

Proof. Using formula (1), we have

$$
\chi_{k}(x)=\chi_{k}\left(x_{1} \vee \cdots \vee x_{m}\right)=\sum_{\substack{S \subseteq[m] \\ S \neq \emptyset}}(-1)^{|S|-1} \chi_{k}\left(\bigwedge_{i \in S} x_{i}\right) .
$$

Observe that, for every $S \subseteq[m],|S| \geq 2, \bigwedge_{i \in S} x_{i}$ can be expressed as the join of terms of the form $x_{i, t} \wedge x_{j, s}$, whose generalized characteristic $\chi_{k}$ is equal to 0 . So, applying again formula (1), it is immediately seen that, in these cases, $\chi_{k}\left(\bigwedge_{i \in S} x_{i}\right)=0$ and then $\chi_{k}(x)=\chi_{k}\left(x_{1}\right)+\cdots+\chi_{k}\left(x_{m}\right)$. Finally, the claim follows applying Proposition 3.23.

Proposition 3.25. Every element $x$ of $\mathcal{D}$ can be written as $x=\left(x_{1} \vee \cdots \vee\right.$ $\left.x_{h}\right) \vee\left(x_{h+1} \vee \cdots \vee x_{m}\right)$ where 
1. $x_{i}=x_{i, 1} \vee \cdots \vee x_{i, \ell_{i}}$ is a join of join-irreducibles with $r_{\widehat{P}}\left(x_{i, t} \wedge x_{i, t+1}\right) \geq k$ for every $t=1, \ldots, \ell_{i}-1$, and $r_{\widehat{P}}\left(x_{i, t} \wedge x_{j, s}\right)<k$ for $t=1, \ldots, \ell_{i}$, $s=1, \ldots, \ell_{j}$, whenever $i \neq j$, for every $i, j=1, \ldots, h$;

2. $x_{i}$ is a join-irreducible with $r_{\widehat{P}}\left(x_{i}\right)<k$, for every $i=h+1, \ldots, m$.

Proof. Let $x=p_{1} \vee \cdots \vee p_{s}$, where $\left(p_{1}, \ldots, p_{s}\right)$ is a Dyck-like antichain. For the first element $p_{1}$ there are two possible cases. If $r_{\widehat{P}}\left(p_{1}\right)<k$, then $p_{1}$ is one of the $x_{i}$ 's. Otherwise, if $r_{\widehat{P}}\left(p_{1}\right) \geq k$, then consider the first index $i$ such that $r_{\widehat{P}}\left(p_{i} \wedge p_{i+1}\right)<k$ : then $p_{1} \vee \cdots \vee p_{i}$ is a join of join-irreducibles such that $r_{\widehat{P}}\left(p_{j} \wedge p_{j+1}\right) \geq k$, for $j<i$, and so it is one of the $x_{i}$ 's. Repeating this argument and rearranging the $x_{i}$ in the correct order, we obtain the desired decomposition.

Any decomposition of the kind described in Proposition 3.25 will be said a $k$-quasi-join-irreducible decomposition of the element $x \in \mathcal{D}$, and the elements $x_{1}, \ldots, x_{h}$ appearing in such a decomposition will be called $k$-quasi-join-irreducibles.

Theorem 3.26. The generalized characteristic $\chi_{k}(x)$ of an element $x \in$ $\mathcal{D}$ is equal to the number of $k$-quasi-join-irreducibles in any $k$-quasi-joinirreducible decomposition of $x$.

Proof. By Proposition 3.25, every element $x$ of $\mathcal{D}$ admits a $k$-quasi-joinirreducible decomposition $x=x_{1} \vee \cdots \vee x_{h} \vee x_{h+1} \vee \cdots \vee x_{m}$. Applying formula (1) we have

$$
\chi_{k}(x)=\sum_{\substack{S \subseteq[m] \\ S \neq \emptyset}}(-1)^{|S|-1} \chi_{k}\left(\bigwedge_{i \in S} x_{i}\right)
$$

If $S$ contains an $i$ such that $r_{\widehat{P}}\left(x_{i}\right)<k$ then $\chi_{k}\left(\bigwedge_{i \in S} x_{i}\right)=0$. Therefore in the computation of $\chi_{k}(x)$ all the join-irreducibles with rank strictly less than $k$ in $\widehat{P}$ can be discarded, i.e.

$$
\chi_{k}(x)=\sum_{\substack{S \subseteq[h] \\ S \neq \emptyset}}(-1)^{|S|-1} \chi_{k}\left(\bigwedge_{i \in S} x_{i}\right)=\chi_{k}\left(x_{1} \vee \cdots \vee x_{h}\right)
$$

Finally, the claim follows from Proposition 3.24.

As a consequence of Theorem 3.26 it follows that the number of $k$ quasi-join-irreducibles in any $k$-quasi-join-irreducible decomposition of an element $x$ is constant. Moreover, from Theorem 3.26 and Proposition 3.10, 

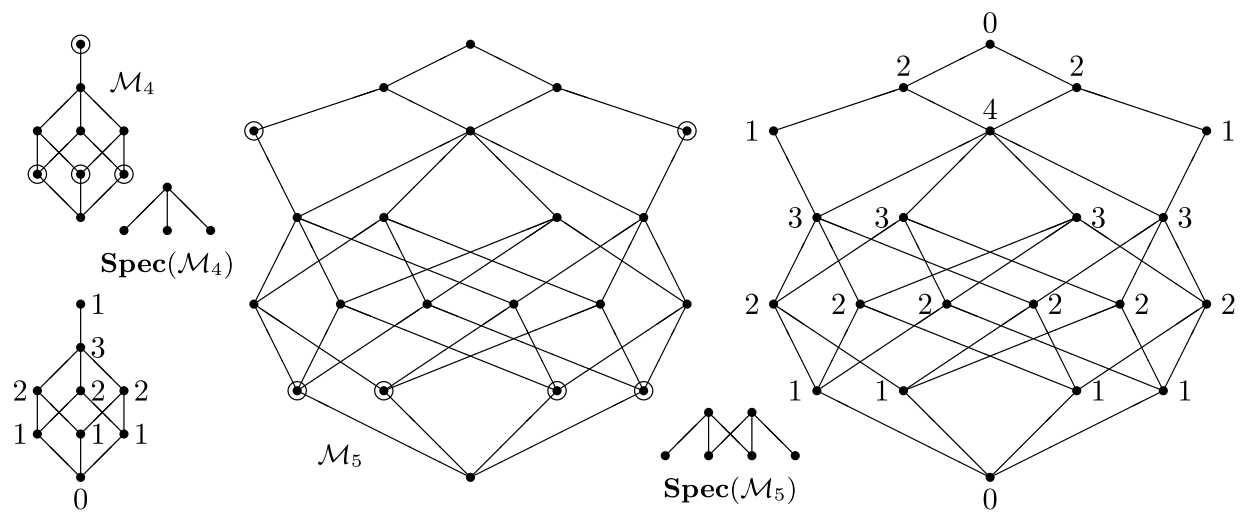

Figure 5: The Motzkin lattices $\mathcal{M}_{4}$ and $\mathcal{M}_{5}$, their spectra and the distribution of the characteristic.

we have the following interpretation of the generalized characteristics of Dyck lattices.

Theorem 3.27. The generalized characteristic $\chi_{k}(x)$ of an element $x$ of the Dyck lattice $\mathcal{D}_{n}$ is equal to the number of k-tunnels of $x$.

\section{Motzkin lattices}

\subsection{Representation}

The Motzkin lattice $\mathcal{M}_{n}$ is a distributive lattice of height $\left\lfloor\frac{n}{2}\right\rfloor\left\lceil\frac{n}{2}\right\rceil$, with minimum $H^{n}$, and maximum $U^{k} D^{k}$ when $n=2 k$ or $U^{k} H D^{k}$ when $n=$ $2 k+1$, with $n-1$ atoms of the form $H^{k} U D H^{n-k-2}$ and just one coatom $U^{k-1} H D^{k-1}$ when $n=2 k$ and two coatoms $U^{k-1} H U D^{k}$ and $U^{k} D H D^{k-1}$ when $n=2 k+1$, It is easy to see that the socle is the path $s=U H^{n-2} D$ and that the principal ideal $\downarrow s$ is isomorphic to a Boolean algebra $B_{n-1}$ while the principal filter $\uparrow s$ is isomorphic to a Motzkin lattice $\mathcal{M}_{n-2}$, whenever $n \geq 1$ (see Figure 5). The rank function is given by the area determined by the path, i.e. $r(x)=\mathcal{A}(x)$.

Proposition 4.1. The join-irreducibles of the Motzkin lattice $\mathcal{M}_{n}$ are the paths having the form

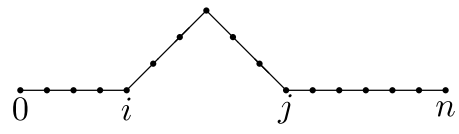


The spectrum of $\mathcal{M}_{n}$ is isomorphic to the poset of the intervals of even length of a chain $\mathcal{C}_{n}$ having $n+1$ elements, i.e. $\operatorname{Spec}\left(\mathcal{M}_{n}\right) \simeq\left\{(i, j) \in \mathcal{C}_{n}^{2}: \exists k \in\right.$ $\mathbb{N}$ such that $j-i=2 k+2\}$.

Proof. If $x \in \mathcal{M}_{n}$ has an horizontal step at height $>0$, then it is easy to see that $x$ can be obtained as the join of two smaller paths. Hence a joinirreducible cannot have horizontal steps at height $>0$. If $x$ had more than one peak, then it could be expressed as the join of all paths obtained from $x$ by replacing each peak with a couple of horizontal steps, one peak at a time. So $x$ can have only one peak.

Remark. Motzkin paths are not Dyck-like paths, nevertheless the lattice $\mathcal{M}_{n}$ of Motzkin paths of length $n$ is a Dyck-like lattice. Notice that $\mathcal{M}_{n}$ is isomorphic to the lattice of Dyck paths of length $2 n$ without three consecutive down steps [4]. This agrees with our previous conjecture on the representation of Dyck-like lattices.

Motzkin lattices are not special Dyck-like lattices. For instance, in $\mathcal{M}_{5}$ the meet of the paths $U^{2} D^{2} H$ and $H U^{2} D^{2}$ is $H U H D H$, which is different from the minimum $\widehat{0}$ and not join-irreducible. However, we can prove a result similar to Proposition 3.10 for the meet of two join-irreducibles in $\mathcal{M}_{n}$, which will allow to compute the Euler characteristic also in this case.

\subsection{Characteristic}

To give a combinatorial description of the characteristic for Motzkin lattices we cannot use the theory developed in the previous sections, since in a Motzkin lattice it could happen that the meet of two join-irreducibles is neither $\widehat{0}$ nor a join-irreducible, as we have seen in Section 4.1. However, the arguments developed for Dyck paths can be adapted to the Motzkin case and lead to analogous results.

A truncated pyramid of a Motzkin path $x$ is a sequence of $k \geq 1$ up steps followed by a sequence of $m \geq 1$ horizontal steps followed by a sequence of $k$ down steps, i.e. $U^{k} H^{m} D^{k}$. The positive integer $k$ is called the dimension of the truncated pyramid, whereas $m$ is its length. Moreover, we say that a truncated pyramid has height $h$ when the sequence of horizontal steps lies on the line $y=h$. In the sequel, we will denote by $T_{n, m, k}$ the set of Motzkin paths of length $n$ having only horizontal steps at height 0 , except for a unique truncated pyramid of dimension $k$ and length $m$. An element of $T_{n, m, k}$ will be called a Motzkin path with a unique truncated pyramid of length $m$ and dimension $k$ (see Figure 6). 


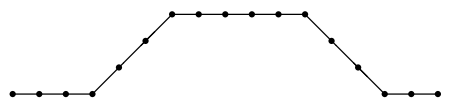

(a)

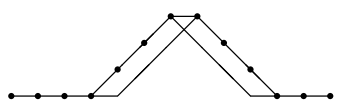

(b)

Figure 6: (a) A Motzkin path in $T_{16,5,3}$. (b) A Motzkin path in $T_{12,1,3}$ and the two join-irreducibles it covers.

Now we are ready to state our result on the meet of two join-irreducible Motzkin paths.

Proposition 4.2. In $\mathcal{M}_{n}$ the meet of two join-irreducibles is either $\widehat{0}$ or a join-irreducible or an element of $T_{n, 1, k}, k \leq n-2$ (i.e., a Motzkin path with a unique truncated pyramid of length 1$)$.

Proof. Two join-irreducible Motzkin paths that do not meet at $\widehat{0}$ must intersect at a point $\left(x_{0}, y_{0}\right)$ with $y_{0}>0$. Then, their meet is a join-irreducible path if $y_{0} \in \mathbb{N}$, whereas it belongs to $T_{n, 1, k}$ if $y_{0} \notin \mathbb{N}$.

Remark. The above proof can be reformulated in a bijective fashion as follows. Let $E_{n}$ be the set obtained by taking all join-irreducibles of $\mathcal{M}_{n}$ and all elements of $T_{n, 1, h}$, for $h=1,2, \ldots, n-2$. $E_{n}$ with the induced order is isomorphic to $\operatorname{Spec}\left(\mathcal{D}_{n}\right)$. Indeed, in $E_{n}$ each element of $T_{n, 1, h}$ covers precisely two join-irreducibles (see Figure 6(b)) and each join-irreducible covers precisely two elements of $T_{n, 1, h}$, for a suitable $h$. Alternatively, map each element of $\operatorname{Spec}\left(\mathcal{D}_{n}\right)$ to the element of $E_{n}$ obtained by performing the following substitutions, when reading the path from left to right: $U U \rightarrow U$, $U D \rightarrow H, D D \rightarrow D$. The resulting map is an isomorphism, which maps $(U D)^{i} U^{k} D^{k}(U D)^{j}$ into $H^{i} U^{\left\lfloor\frac{k}{2}\right\rfloor} H^{k-2\left\lfloor\frac{k}{2}\right\rfloor} D^{\left\lfloor\frac{k}{2}\right\rfloor} H^{j}$.

First of all, we compute the characteristic of some particular Motzkin paths.

Lemma 4.3. If $x \in T_{n, m, h}$, then $\chi(x)=(-1)^{h+1} m+1$.

Proof. Since a Motzkin path $x \in T_{n, 1,1}$ is the join of two join-irreducibles whose meet is $\widehat{0}$, we have $\chi(x)=2$. Similarly, since a Motzkin path $x \in T_{n, 1,2}$ is the join of two join-irreducibles whose meet is a Motzkin path belonging to $T_{n, 1,1}$, we have $\chi(x)=1+1-2=0$. Iterating this argument it follows that for every $x \in T_{n, 1, h}$ the characteristic is $\chi(x)=2$ when $h$ is odd and $\chi(x)=0$ when $h$ is even, i.e. $\chi(x)=(-1)^{h+1}+1$. We now proceed by induction on the length $m$. If $x \in T_{n, m+1, h}$, then $x=H^{a} U^{h} H^{m+1} D^{h} H^{b}$ and hence $x=x_{1} \vee x_{2}$ where $x_{1}=H^{a} U^{h} H^{m} D^{h} H^{b+1}, x_{2}=H^{a+1} U^{h} H^{m} D^{h} H^{b}$. 
Since $x_{1} \wedge x_{2}=H^{a+1} U^{h} H^{m-1} D^{h} H^{b+1} \in T_{n, m-1, h}$, we have

$$
\begin{aligned}
\chi(x) & =\chi\left(x_{1}\right)+\chi\left(x_{2}\right)-\chi\left(x_{1} \wedge x_{2}\right) \\
& =(-1)^{h+1} m+1+(-1)^{h+1} m+1-(-1)^{h+1}(m-1)-1
\end{aligned}
$$

that is $\chi(x)=(-1)^{h+1}(m+1)+1$. So, the lemma is proved.

Remark. The maximum $\widehat{1}$ of $\mathcal{M}_{n}$ is join-irreducible when $n=2 k$ and belongs to $T_{2 k+1,1, k}$ when $n=2 k+1$. Hence, from Lemma 4.3, it follows that $\chi\left(\mathcal{M}_{n}\right)=1$ when $n$ is even, $\chi\left(\mathcal{M}_{n}\right)=0$ when $n=4 k+1$ and $\chi\left(\mathcal{M}_{n}\right)=2$ when $n=4 k+3$.

In any Motzkin lattice each quasi-join-irreducible has a particular joinirreducible decomposition, coming directly from the definition of quasi-joinirreducible element. However, for our purposes, another kind of decomposition will be useful in representing quasi-join-irreducibles. In fact, every quasi-join-irreducible element $x \in \mathcal{M}_{n}$ can be expressed, in a unique way, as $x=s_{1} \vee \cdots \vee s_{k}$, where each $s_{i}$ is either join-irreducible or it belongs to $T_{n, m, h}$, and $s_{i} \wedge s_{i+1} \neq \widehat{0}$, for every $i<k$. To see this, just write $x=$ $t_{1} \vee \cdots \vee t_{r}$ as a join of join-irreducibles and group together all the consecutive join-irreducibles whose join gives rise to a Motzkin path belonging to some $T_{n, m, h}$. Such a decomposition will be called the Motzkin decomposition of the quasi-join-irreducible $x$.

Now, we are ready to state and proof the fundamental step in the determination (and combinatorial interpretation) of the characteristic of $\mathcal{M}_{n}$. Our main proposition will be preceded by a technical lemma.

Lemma 4.4. Let $x \in \mathcal{M}_{n}$ be a quasi-join-irreducible element and $x=$ $s_{1} \vee \cdots \vee s_{k}$ its Motzkin decomposition. Then, for every $i<k, s_{i} \wedge s_{i+1}$ is join-irreducible.

Proof. If $s_{i}$ and $s_{i+1}$ are both join-irreducibles, the conclusion follows from the definition of Motzkin decomposition (since otherwise $s_{i}$ and $s_{i+1}$ would "contribute" to a truncated pyramid in the Motzkin decomposition of $x$ ). If at least one of the two is an element of some $T_{n, m, h}$, then $s_{i} \wedge s_{i+1}$ is equal to the meet of two join-irreducibles. Indeed, if $s_{i}$ is join-irreducible and $s_{i+1} \in$ $T_{n, m, h}$, then $s_{i} \wedge s_{i+1}=s_{i} \wedge x$, where $s_{i+1}=x \vee x_{1} \vee \cdots \vee x_{r}$ is a join-irreducible decomposition of $s_{i+1}$ and $x$ is the join-irreducible having minimum abscissa; then an argument similar to the above one shows that $s \wedge x$ is join-irreducible. The remaining cases can be dealt with in a similar way.

Let $x \in \mathcal{M}_{n}$. We will write $o(x)$ for the number of horizontal steps at odd height and $e(x)$ for the number of horizontal steps at even nonzero height (i.e. at even height and not lying on the $x$-axis). 
Proposition 4.5. Let $x \in \mathcal{M}_{n}$ be a quasi-join-irreducible. Then $\chi(x)=$ $o(x)-e(x)+1$.

Proof. Let $x=s_{1} \vee \cdots \vee s_{k}$ be the Motzkin decomposition of $x$. From formula (1) we have

$$
\chi(x)=\chi\left(s_{1} \vee \cdots \vee s_{k}\right)=\sum_{\substack{S \subseteq[k] \\ S \neq \emptyset}}(-1)^{|S|-1} \chi\left(\bigwedge_{i \in S} s_{i}\right) .
$$

Since $\mathcal{M}_{n}$ is a Dyck-like lattice, we can proceed as in Proposition 3.16, noticing that a result analogous to Lemma 3.13 holds for the Motzkin decomposition. We first observe that, if $\min S=\max S$, then the contribution to the sum is $\chi\left(s_{1}\right)+\cdots+\chi\left(s_{k}\right)$. Some of the $s_{i}$ 's are join-irreducibles (and so their contribution is 1 ), but some of them could be paths in $T_{n, m, h}$. If $s_{i} \in T_{n, m, h}$, then from Lemma 4.3 it follows that $\chi\left(s_{i}\right)=(-1)^{h+1} m+1$. Starting from this remark, it is not difficult to show that $\chi\left(s_{1}\right)+\cdots+\chi\left(s_{k}\right)=$ $k+o(x)-e(x)$. Now, if $\max S=\min S+1$, the contribution to the sum is $-\sum_{i<k} \chi\left(s_{i} \wedge s_{i+1}\right)=-(k-1)$, since all meets $s_{i} \wedge s_{i+1}$ are necessarily join-irreducible by Lemma 4.4. Finally, if $\max S=\min S+r$, with $r>1$, using an argument completely analogous to the one used in the Dyck case, we find that the contribution of these subsets to the sum in (5) is zero. So, in conclusion, we have $\chi(x)=k+o(x)-e(x)-k+1=$ $o(x)-e(x)+1$.

Since Motzkin lattices are Dyck-like, from Proposition 3.17 it follows that every element of a Motzkin lattice has a quasi-join-irreducible decomposition. Let $\|x\|$ be the number of all quasi-join-irreducibles in a decomposition of $x \in \mathcal{M}_{n}$, and let $o^{\prime}(x)$ be the number of horizontal steps at odd height different from 1.

Theorem 4.6. The characteristic of a Motzkin path $x \neq \widehat{0}$ is $\chi(x)=\|x\|+$ $o^{\prime}(x)-e(x)$.

Proof. If $x=x_{1} \vee \cdots \vee x_{k}$ is a quasi-join-irreducible decomposition of $x$, then $\chi(x)=\chi\left(x_{1}\right)+\cdots+\chi\left(x_{k}\right)=o\left(x_{1}\right)-e\left(x_{1}\right)+1+\cdots+o\left(x_{k}\right)-e\left(x_{k}\right)+1=$ $o^{\prime}(x)-e(x)+k$.

To properly state our last result of the present section, we characterize the quasi-join-irreducible elements of Motzkin lattices, thus obtaining an analog of Proposition 3.21. The easy proof is left to the reader.

Proposition 4.7. A Motzkin path is quasi-join-irreducible if and only if either it has a unique elevated factor having no horizontal steps at height 1 or it has a unique peak at height 1. 
A reverse truncated pyramid of height $h$ is any factor of a Motzkin path of the form $D H^{k} U$, where $k \geq 1$ and the sequence $H^{k}$ of horizontal steps lies on the line $y=h$. The height of a peak is given by its ordinate. If $f_{h}(x), p_{h}(x)$, $t_{h}(x)$ and $r_{h}(x)$ are respectively the number of truncated pyramids, peaks, maximal sequences of consecutive 1-tunnels and reverse truncated pyramid of height $h$ in $x$, then Theorem 4.6 can be interpreted combinatorially as stated in

Theorem 4.8. The characteristic of a Motzkin path $x \in \mathcal{M}_{n}$ is

$$
\chi(x)=o(x)-e(x)+t_{1}(x)+f_{1}(x)+p_{1}(x)-r_{1}(x) .
$$

Proof. We have only to give a combinatorial interpretation of the term $\|x\|$. By virtue of Proposition 4.7, the quasi-join-irreducibles in a decomposition of a Motzkin path $x$ can be of two types only. They can be Motzkin paths with a unique elevated factor having no horizontal steps at height 1 , whose number is equal to the number of maximal sequences of consecutive 1-tunnels of $x$. Otherwise they can be Motzkin paths having a unique peak at height 1 . In this case, we have an isolated peak in $x$, or a sequence of consecutive peaks whose abscissas differ by 1 . In this last case, what we see in $x$ is a sequence of horizontal steps at height 1 . We have three possible configurations:

a) a truncated pyramid at height $1, U H^{m} D$, obtained as the join of $m+1$ peaks at height 1 ,

b) either $U H^{m} U$ or $D H^{m} D$ : in both cases, the configuration is obtained as the join of $m$ peaks at height 1 ,

c) a reverse truncated pyramid $D H^{m} U$ at height 1 obtained as the join of $m-1$ peaks at height 1 .

Hence $\|x\|=t_{1}(x)+p_{1}(x)+h_{1}(x)+f_{1}(x)-r_{1}(x)$, where $h_{1}(x)$ is the number of horizontal steps of $x$ at height 1.

\section{Schröder lattices}

\subsection{Representation}

The Schröder lattice $\mathcal{S}_{n}$ is a distributive lattice of height $n^{2}$, with minimum $H^{2 n}$ and maximum $U^{n} D^{n}$, with $n-1$ atoms of the form $H^{2 k} U D H^{2 n-2 k-2}$ and one coatom $U^{n-1} H^{2} D^{n-1}$. The socle is the path $s=(U D)^{n}$ and the principal ideal $\downarrow s$ is isomorphic to a Boolean algebra $B_{n}$. This time, however, the principal filter $\uparrow s$ just contains an isomorphic copy of the Schröder lattice $\mathcal{S}_{n-1}$ consisting of the principal filter generated by the path $s^{\prime}=$ $U H^{2 n-2} D$. Moreover, the interval $\left[s, s^{\prime}\right]$ is a Boolean algebra $B_{n-2}$ (see Figure 7). The rank function is given by the area under the path (as for Motzkin lattices). 

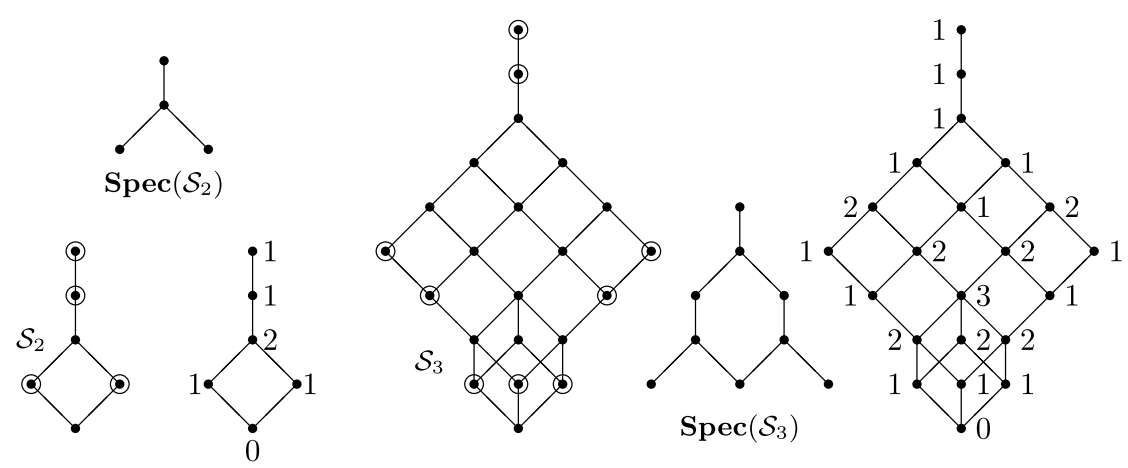

Figure 7: The Schröder lattices $\mathcal{S}_{2}$ and $\mathcal{S}_{3}$, their spectra and the distribution of the characteristic.

Proposition 5.1. The join-irreducibles of the Schröder lattice $\mathcal{S}_{n}$ are the paths of the form

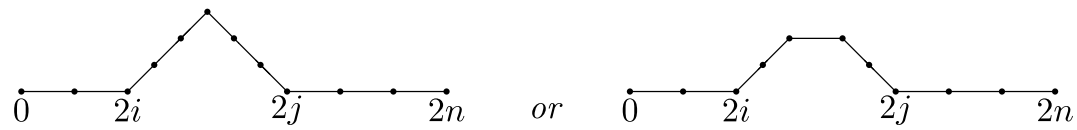

In particular, $\operatorname{Spec}\left(\mathcal{S}_{n}\right) \simeq\left\{(i, j, k) \in \mathcal{C}_{n}^{2} \times \mathcal{C}_{1}: j-i \geq k+1\right\}$.

Proof. Given a Schröder path, there are only two possible ways of getting a path which is covered by the starting one: either replace an occurrence of $U D$ with a double horizontal step or replace a double horizontal step with $D U$.

There are at least two further ways of describing the poset $\operatorname{Spec}\left(\mathcal{S}_{n}\right)$. They are essentially equivalent, but the first one is expressed in purely algebraic language whereas the second one can be considered a sort of combinatorial interpretation.

1. The lexicographic product $P \circ Q$ of two posets $P$ and $Q$ is the set $P \times Q$ endowed with the order defined by setting $\left(x_{1}, y_{1}\right) \leq\left(x_{2}, y_{2}\right)$ when $x_{1}<x_{2}$ or $x_{1}=x_{2}$ and $y_{1} \leq y_{2}$. Then $\operatorname{Spec}\left(\mathcal{S}_{n}\right)$ is isomorphic to the poset obtained by $\operatorname{Spec}\left(\mathcal{D}_{n+1}\right) \circ \mathcal{C}_{1}$ when all the minimal elements are removed (see Figure 7).

2. Denote by $\overrightarrow{\operatorname{Int}}\left(\mathcal{C}_{n}\right)$ the set of oriented intervals of $\mathcal{C}_{n}$. An interval $I$ of a poset $P$ is said to be oriented upward (downward) when its elements are listed in such a way that, if $x<y$ in $P$, then $x$ precedes (follows) $y$ in the above listing (in this way $I$ is not just a set but, more precisely, an ordered $t$-uple). If $P$ is a chain, $P=\mathcal{C}_{n}$, then its intervals can 
have only two orientations, say negative (from top to bottom) and positive (from bottom to top). We can introduce a partial order on $\overrightarrow{\mathbf{I n t}}\left(\mathcal{C}_{n}\right)$ by simply declaring that, for any $I, J \in \overrightarrow{\mathbf{I n t}}\left(\mathcal{C}_{n}\right), I \leq J$ when either $I$ is contained in $J$ as ordinary intervals or $I=J$ as ordinary intervals but $I$ is negative and $J$ is positive. It is not difficult to see that, endowed with this partial order, $\overrightarrow{\operatorname{Int}}\left(\mathcal{C}_{n-1}\right) \simeq \operatorname{Spec}\left(\mathcal{S}_{n}\right)$ (observe that, in $\overrightarrow{\operatorname{Int}}\left(\mathcal{C}_{n}\right)$, as far as singleton intervals are concerned, there is no distinction between positive and negative intervals).

Proposition 5.2. The Schröder lattices $\mathcal{S}_{n}$ are special Dyck-like lattices where, in particular, the meet of any two join-irreducibles is $\widehat{0}$ or a joinirreducible having a unique peak.

Proof. As we did for Dyck paths, label each join-irreducible of $\mathcal{S}_{n}$ with the abscissa of its unique (truncated) pyramid: this labelling satisfies the condition in the definition of a Dyck-like lattice. Since two Schröder paths of the same length can cross only at points with integer coordinates and cannot cross in the middle point of a double horizontal step of one of them, if the intersection of two join-irreducibles is not the minimum $\widehat{0}$, then it is necessarily a join-irreducible with a unique peak.

\subsection{Characteristic}

The characteristic of Schröder lattices admits a combinatorial interpretation analogous to the one given for Dyck lattices. Indeed, following the same lines of the proofs of Proposition 3.21 and Corollary 3.22 and using Proposition 5.1, we have

Theorem 5.3. A Schröder path is quasi-join-irreducible if and only if it has exactly one 0-tunnel.

Theorem 5.4. The characteristic of a Schröder path equals the number of its 0-tunnels.

\section{Final remarks on rank unimodality}

An interesting property common to several sequences arising in combinatorics is unimodality. Specifically, a sequence $\left\{a_{0}, a_{1}, \ldots, a_{n}\right\}$ of positive integers is unimodal when there exists an index $k$ such that $a_{0} \leq a_{1} \leq \cdots \leq$ $a_{k} \geq a_{k+1} \geq \cdots \geq a_{n}$, and a polynomial is unimodal when the sequence of its coefficients is unimodal. In the case of ranked posets this is often a property of the distribution of the elements of given rank. More precisely, a (finite) poset $P$ is rank unimodal when it is ranked and its rank poly- 
nomial (or equivalently the sequence of its Whitney numbers) is unimodal. The Whitney number $W_{k}(P)$ is the number of all elements of $P$ with rank $k$ whereas the rank polynomial is $R(P ; q)=W_{0}(P)+W_{1}(P) q+\cdots+W_{h}(P) q^{h}$, where $h=r(P)$ is the height of $P$.

Dyck lattices. Let $D_{n}(q)$ be the rank polynomial of $\mathcal{D}_{n}$. Since every nonempty Dyck path decomposes as $U \gamma^{\prime} D \gamma^{\prime \prime}$ (where $\gamma^{\prime}$ and $\gamma^{\prime \prime}$ are arbitrary Dyck paths), we have the recurrence

$$
D_{n+1}(q)=\sum_{k=0}^{n} q^{k} D_{k}(q) D_{n-k}(q)
$$

with the initial condition $D_{0}(q)=1$. It is easy to see that the generating series for these polynomials satisfies the identity $D(q, t)=(1-t D(q, q t))^{-1}$ from which it is possible to obtain an expansion as a continued fraction [23]. The polynomials $D_{n}(q)$ define a $q$-analog of Catalan numbers, namely $q^{\left(\begin{array}{c}n \\ 2\end{array}\right)} D_{n}(1 / q)=C_{n}(q)$, where the $C_{n}(q)$ 's are the $q$-Catalan numbers defined as the sum $\sum_{x} q^{\mathcal{A}(x)}$ over all lattice paths from $(0,0)$ to $(n, n)$, with steps $(1,0)$ and $(0,1)$, never rising above the line $y=x$, where $\mathcal{A}(x)$ is the area of the region determined by the path and the $x$-axis [14] [23, p. 235]. The Whitney numbers of $\mathcal{D}_{n}$ appears in [21] (essentially) as sequence A129182. It is still an open problem [6] to prove or disprove that the rank polynomials $D_{n}(q)$ are unimodal for every $n \in \mathbb{N}$. This problem is also mentioned in [24], where it is conjectured that the Young's lattices associated with the staircase partitions $(n, n-1, \ldots, 2,1)$ are rank unimodal.

Motzkin lattices. Let $M_{n}(q)$ be the rank polynomial of $\mathcal{M}_{n}$. Since every non-empty Motzkin path decomposes as $H \gamma$ or $U \gamma^{\prime} D \gamma^{\prime \prime}$ (where $\gamma, \gamma^{\prime}$ and $\gamma^{\prime \prime}$ are arbitrary Motzkin paths), we have the recurrence

$$
M_{n+2}(q)=M_{n+1}(q)+\sum_{k=0}^{n} q^{k+1} M_{k}(q) M_{n-k}(q)
$$

with the initial conditions $M_{0}(q)=M_{1}(q)=1$. Their generating series $M(q, t)$ satisfies the identity $M(q, t)=\left(1-t-q t^{2} M(q, q t)\right)^{-1}$ and hence admits an expansion as a continued fraction. The Whitney numbers of $\mathcal{M}_{n}$ appear in [21] as sequence A129181 and also in this case we can conjecture that the lattices $\mathcal{M}_{n}$ are rank-unimodal.

Schröder lattices. Let $S_{n}(q)$ be the rank polynomial of $\mathcal{S}_{n}$. Since every nonempty Schröder path decomposes as $H H \gamma$ or $U \gamma^{\prime} D \gamma^{\prime \prime}$ (where $\gamma, \gamma^{\prime}$ and $\gamma^{\prime \prime}$ 
are arbitrary Schröder paths), we have the recurrence

$$
S_{n+1}(q)=S_{n}(q)+\sum_{k=0}^{n} q^{2 k+1} S_{k}(q) S_{n-k}(q)
$$

with the initial condition $S_{0}(q)=1$. Their generating series $S(q, x)$ satisfies the identity $S(q, x)=\left(1-x-q x S\left(q, q^{2} x\right)\right)^{-1}$ and hence also this time it has an expansion as a continued fraction. The Whitney numbers of $\mathcal{S}_{n}$ appear in [21] as sequence A129179. Also in this case it is still an open problem [6] to prove the rank-unimodality of the lattices $\mathcal{S}_{n}$.

\section{Acknowledgment}

The authors would like to express their gratitude to an anonymous referee for many constructive comments which have improved both the content and the presentation of this paper.

\section{References}

[1] M. Aigner, Combinatorial Theory, Springer-Verlag, New York, 1979. MR0542445.

[2] C. Banderier, P. Flajolet, Basic analytic combinatorics of directed lattice paths, Theoret. Comput. Sci. 281 (2002), 37-80. MR1909568.

[3] E. Barcucci, A. Bernini, L. Ferrari, M. Poneti, A distributive lattice structure connecting Dyck paths, noncrossing partitions and 312-avoiding permutations, Order 22 (2005), 311-328. MR2220342.

[4] A. Bernini, L. Ferrari, Order properties of the Motzkin and Schröder families, Australasian J. Combin. 39 (2007), 259-272. MR2351206.

[5] G. Birkhoff, Lattice Theory, third edition, Amer. Math. Soc. Colloquium Publications, Providence, 1966.

[6] J. Bonin, L. Shapiro, R. Simion, Some q-analogues of the Schröder numbers arising from combinatorial statistics on lattice paths, J. Statist. Plann. Inference 34 (1993), 35-55. MR1209988.

[7] P. Crawley, R. P. Dilworth, Algebraic Theory of Lattices, Prentice-Hall, N.J., 1973.

[8] B. A. Davey, H. A. Priestley, Introduction to Lattices and Order, Cambridge University Press, New York, 2002. MR1902334. 
[9] P. Duchon, On the enumeration and generation of generalized Dyck words, Discrete Math. 255 (2000), 121-135. MR1798327.

[10] S. Elizalde, Fixed points and excedances in restricted permutations, Proceedings of FPSAC, 2003.

[11] S. Elizalde, Multiple pattern-avoidance with respect to fixed points and excedances, Electron. J. Combin. 11 (2004), \#R51 (40pp.). MR2097317.

[12] L. Ferrari, Some combinatorics related to central binomial coefficients: Grand-Dyck paths, coloured noncrossing partitions and signed pattern avoiding permutations, Graphs and Combinatorics 26 (2010), 51-70. MR2606618.

[13] L. Ferrari, R. Pinzani, Lattices of lattice paths, J. Statist. Plann. Inference 135 (2005), 77-92. MR2202340.

[14] J. Fürlinger, J. Hofbauer, q-Catalan numbers, J. Combin. Theory Ser. A 40 (1985), 248-264. MR0814413.

[15] D. A. Klain, G.-C. Rota, Introduction to Geometric Probability, Cambridge University Press, New York, 1997. MR1608265.

[16] V. Klee, The Euler characteristic in combinatorial geometry, Amer. Math. Monthly 79 (1963), 119-127. MR0146101.

[17] E. Munarini, On the Euler characteristic of finite distributive lattices, pp. 165-186, in From Combinatorics to Philosophy. The Legacy of G.C. Rota, E. Damiani, O. D'Antona, V. Marra, F. Palombi, editors, Springer, 2009.

[18] G.-C. Rota, On the Combinatorics of the Euler Characteristic, Studies in Pure Mathematics, Academic Press, London, 1971, pp. 221-233. MR0282892.

[19] L. Santocanale, On the join dependency relation in multinomial lattices, Order 24 (2007), 155-179. MR2358079.

[20] A. Sapounakis, I. Tasoulas, P. Tsikouras, On the dominance partial ordering of Dyck paths, J. Integer Seq. 9 (2006), \#06.2.5 (17 pp.). MR2217231.

[21] N. J. A. Sloane, On-Line Encyclopedia of Integer Sequences, published electronically at http://oeis.org.

[22] R. P. Stanley, The Fibonacci lattice, Fibonacci Quart. 13 (1975), 215232. MR0387143. 
[23] R. P. Stanley, Enumerative Combinatorics, Volume 2, Cambridge Studies in Advanced Mathematics 62, Cambridge University Press, Cambridge, 1999. MR1676282.

[24] D. Stanton, Unimodality and Young's lattice, J. Combin. Theory Ser. A 54 (1990), 41-53. MR1051777.

LUCA FERRARI

Dipartimento Di Sistemi e Informatica

Universitá Di FiRENZE

Viale Morgagni 65, 50134 Firenze

ITALY

E-mail address: ferrari@dsi.unifi.it

EMANuele MunARini

Dipartimento Di MATEMATiCA

Politecnico di Milano

Piazza Leonardo da Vinci 32, 20133 Milano

ITALY

E-mail address: emanuele.munarini@polimi.it

ReCEIVED April 23, 2010 